\title{
Amine-Functionalized ZIF-8 as a Fluorescent Probe for Breath VOC Biomarker Detection of Lung Cancer Patients
}

Yuanhan Xia ${ }^{\dagger, t}$, Yi Hong ${ }^{\dagger, t}$, Rongchuang Geng ${ }^{\S}$, Xue Lit ${ }^{\dagger, t}$, Ailan Qu ${ }^{\|}$, Zhen Zhou ${ }^{\dagger, t}$, Zhijuan Zhang ${ }^{\dagger, t}, s^{*}$

$\dagger$ Institute of Mass Spectrometer and Atmospheric Environment, Jinan University, Guangzhou 510632, China.

$\$$ Guangdong Provincial Engineering Research Center for Online Source Apportionment System of Air Pollution, Guangzhou 510632, China.

§College of Pharmacy, Henan University of Chinese Medicine, Zhengzhou, Henan, 450046.

॥College of Chemistry and Materials Science, Jinan University, Guangzhou, 510632, China. 


\section{Part I:}

\section{Figure S.I. captions:}

Figure S1. Thermogravimetric (TG) profiles of ZIF-8 and modified ZIF-8. (a) ZIF-8 and MMEN samples (b) ZIF-8 and MAEA samples.

Figure S2. The PXRD patterns of the recovered ZIF-8 sample after four cycles.

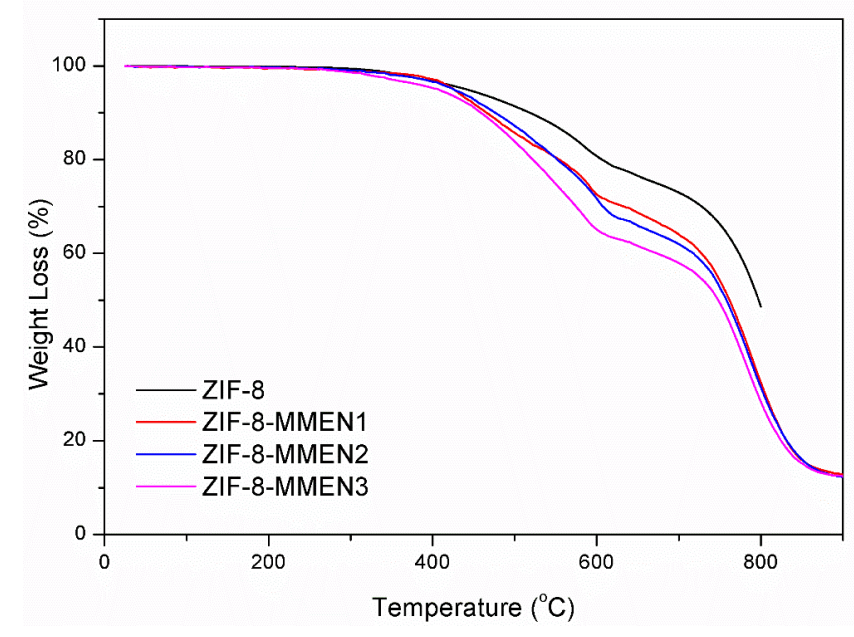

(a)

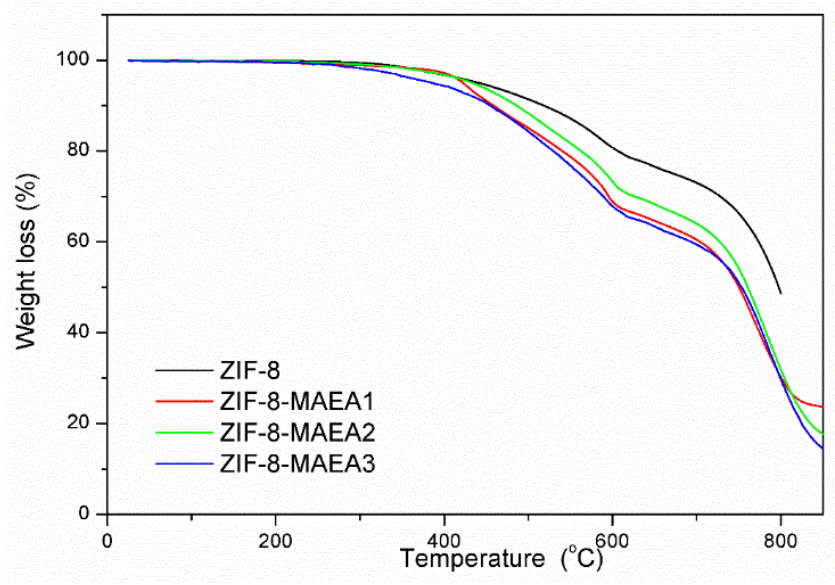

(b)

Figure S1. Thermogravimetric (TG) profiles of ZIF-8 and modified ZIF-8. (a) ZIF-8 and MMEN samples (b) ZIF-8 and MAEA samples. 


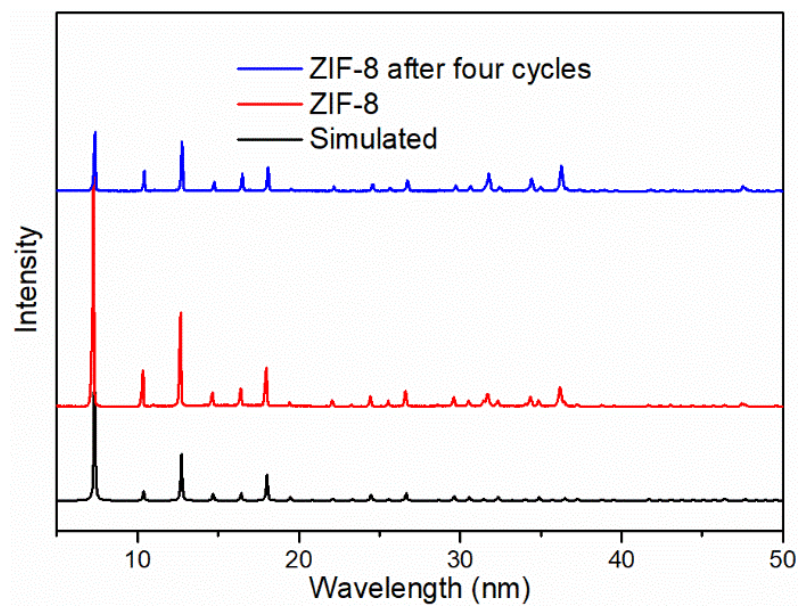

Figure S2. The PXRD patterns of the recovered ZIF-8 sample after four cycles.

Part II :

Table S.I. captions:

Table S1. Concentration gradients of MMEN and MAEA used in the ZIF-8 postmodification process

Table S2. The weight losses of ZIF-8 and ZIF-8@MMEN samples

Table S3. The weight losses of ZIF-8 and ZIF-8@MAEA samples

Table S1 . Concentration gradients of MMEN and MAEA used in the ZIF-8 postmodification process

\begin{tabular}{lll}
\hline Sample & ZIF-8 $(\mathrm{g})$ & $\begin{array}{l}\text { Concentration of MMEN/MAEA } \\
(\mathrm{mol} / \mathrm{L})\end{array}$ \\
\hline ZIF-8-MMEN1/ MAEA1 & 0.5 & $0.046 / 0.046$ \\
ZIF-8-MMEN2/ MAEA2 & 0.5 & $0.438 / 0.436$ \\
ZIF-8-MMEN3/ MAEA3 & 0.5 & $2.297 / 2.289$ \\
\hline
\end{tabular}


Table S2. The weight losses of ZIF-8 and ZIF-8@MMEN samples

\begin{tabular}{lll}
\hline Sample & First step $\left({ }^{\circ} \mathrm{C}\right)$ & Second step $\left({ }^{\circ} \mathrm{C}\right)$ \\
\hline ZIF-8 & $315.23-630.85$ & $630.85-890.00$ \\
ZIF-8-MMEN1 & $356.68-666.84$ & $666.84-885.10$ \\
ZIF-8-MMEN2 & $340.15-659.87$ & $659.87-889.25$ \\
ZIF-8-MMEN3 & $303.97-655.13$ & $655.13-881.50$ \\
\hline
\end{tabular}

Table S3. The weight losses of ZIF-8 and ZIF-8@MAEA samples

\begin{tabular}{lll}
\hline Sample & First step $\left({ }^{\circ} \mathrm{C}\right)$ & Second step $\left({ }^{\circ} \mathrm{C}\right)$ \\
\hline ZIF-8 & $315.23-630.85$ & $630.85-890.00$ \\
ZIF-8-MAEA1 & $315.55-687.21$ & $686.55-891.94$ \\
ZIF-8- MAEA2 & $289.62-649.21$ & $648.54-889.94$ \\
ZIF-8- MAEA3 & $303.76-664.53$ & $664.53-891.59$ \\
\hline
\end{tabular}

